\title{
Threshold-like associations as a function of disturbance
}

\author{
Kimmo Sorjonen ${ }^{\text {Corresp., }}{ }^{,}$Michael Ingre ${ }^{1,2,3}$, Bo Melin ${ }^{1}$ \\ ${ }^{1}$ Department of Clinical Neuroscience, Karolinska Institute, Stockholm, Sweden \\ 2 Department of Psychology, Stockholm University, Stockholm, Sweden \\ 3 Institute for Globally Distributed Open Research and Education (IGDORE), Stockholm, Sweden \\ Corresponding Author: Kimmo Sorjonen \\ Email address: kimmo.sorjonen@ki.se
}

According to the intelligence-creativity threshold hypothesis, there should be a positive association between intelligence and creative potential up to a certain point, the threshold, after which a further increase in intelligence should have no association with creativity. In the present simulation study, the measured intelligence and creativity of virtual subjects were affected by their true abilities as well as a disturbance factor that varied in magnitude between subjects. The results indicate that the hypothesized threshold-like association could be due to some disturbing factor, e.g. low motivation, illness, or linguistic confusion, that varies between individuals and that affects both measured intelligence and measured creativity, especially if the actual association between intelligence and creativity is weak. This, together with previous negative findings, calls the validity of the intelligencecreativity threshold hypothesis into question. 
1 Running head: THRESHOLD HYPOTHESIS

2

3

4

5 Title: Threshold-Like Associations as a Function of Disturbance

6

7 Authors: Kimmo Sorjonen ${ }^{1}$, Michael Ingre ${ }^{1,2,3}$ \& Bo Melin ${ }^{1}$

$8{ }^{1}$ Department of Clinical Neuroscience, Karolinska Institutet, Stockholm, Sweden

$9 \quad 2$ Department of Psychology, Stockholm University, Stockholm, Sweden

$10{ }^{3}$ Institute for Globally Distributed Open Research and Education (IGDORE), Stockholm,

11 Sweden

12

13 Corresponding Author:

14 Name: Kimmo Sorjonen

15 Adress: Division of Psychology, Department of Clinical Neuroscience, Karolinska Institutet, 171

1665 Solna, Sweden

17 Phone: +46-76-878 4236

18 Email: kimmo.sorjonen@ki.se

19 
21 According to the intelligence-creativity threshold hypothesis, there should be a positive

22 association between intelligence and creative potential up to a certain point, the threshold, after

23 which a further increase in intelligence should have no association with creativity. In the present

24 simulation study, the measured intelligence and creativity of virtual subjects were affected by

25 their true abilities as well as a disturbance factor that varied in magnitude between subjects. The

26 results indicate that the hypothesized threshold-like association could be due to some disturbing

27 factor, e.g. low motivation, illness, or linguistic confusion, that varies between individuals and

28 that affects both measured intelligence and measured creativity, especially if the actual

29 association between intelligence and creativity is weak. This, together with previous negative

30 findings, calls the validity of the intelligence-creativity threshold hypothesis into question. 
According to so called threshold hypotheses, the association between a predictor and an outcome is assumed to look different below and above a specific value, the threshold, on the predictor or alternatively on a third variable. It has, for example, been suggested that: (1) A certain minimum level of dominant language proficiency should be achieved before bilingualism can have a positive cognitive effect (Cummins, 1976, 1979); (2) The economic growth in societies brings about an increase in the citizens' economic welfare (a measure combining income inequalities, social, and environmental factors), but only up to a point beyond which a further increase in economic growth may result in a deterioration of the economic welfare (MaxNeef, 1995); (3) A large enough difference in the quality of the territory held by an already mated and a non-mated potential partner may result in a fitness advantage of the bigamous compared to the monogamous choice (Orians, 1969).

According to the intelligence-creativity threshold hypothesis, high intelligence is necessary, but not sufficient, for high creativity (Guilford, 1967). This has led to the prediction that intelligence should have a positive influence on creative potential but only up to a certain level, the threshold is often set at IQ $120(S D=15)$, after which a further increase in intelligence should no longer influence creative potential (Jauk, Benedek, Dunst, \& Neubauer, 2013). This prediction has received some empirical support (e.g. Cho, Nijenhuis, van Vianen, Kim, \& Lee,

51 2010; Fuchs-Beauchamp, Karnes, \& Johnson, 1993; Jauk et al., 2013; Schubert, 1973; Shi,

52 Wang, Yang, Zhang, \& Xu, 2017) although quite a few studies have failed to demonstrate such a

53 threshold effect (e.g. Kim, 2005; Preckel, Holling, \& Wiese, 2006; Runco \& Albert, 1986; Sligh, 54 Conners, \& Roskos-Ewoldsen, 2005). 
As examples of used measures of creativity, Jauk et al. (2013) measured creative potential

56

57

with: (1) Three alternate uses tasks, where participants were asked to come up with as many novel and uncommon uses as possible for a can, a hairdryer, and a knife; (2) Three instances tasks, where participants were asked to come up with as many novel and uncommon solutions as possible to the problems "What can be elastic?", "What can make noise?", "What could one use for quicker locomotion?". The originality of the responses was rated by four students and both number of ideas (= ideational fluency) and originality were used as measures of creative potential. Creative achievement was measured with the Inventory of Creative Activities and Achievements (ICAA), where respondents rate themselves, on a scale from 0 (= "I have never been engaged in this domain") to 10 (= "I have already sold some of my work in this domain"), across the eight domains arts and crafts, creative cooking, literature, music, performing arts, science and engineering, sports, and visual arts. Jauk et al. (2013) found a threshold-like association between intelligence and creative potential but not between intelligence and creative achievement.

When reading the articles about the intelligence-creativity threshold hypothesis mentioned above, for example noticing a few subjects with very low measured IQ $(\approx 60)$ and creative potential in the plots in Jauk et al. (2013), we started to suspect that this threshold-like association between intelligence and creative potential, if it exists, could, at least to some degree, be due to some disturbing factor, for example low motivation, illness, or linguistic confusion, experienced by some study participants. Of course, in a random sample of 300 individuals from the general population you would expect to get a few individuals with intellectual disability (IQ $<70$ ), but we think that it is quite surprising to see this among subjects who have been recruited, as was the case in Jauk et al., through a local newspaper and the university's mailing list. 
78 Imagine, for example, a few friends who read about a study looking for participants who will get

79 paid. They welcome the opportunity to make some extra money and respond. When filling out

80 the questionnaires measuring IQ and creativity they do not put much effort into it, because they

81 are doing this just for the money. When the researchers plot measured IQ and creativity against

82 each other, these friends show up in the lower left corner. The objective of the present simulation

83 study was to investigate the possibility that observed threshold-like associations might be due to

84 the influence of disturbance.

85

Method

87

\section{Simulation}

Using R 3.5.0 statistical software (R Core Team, 2018) a dataset was simulated through the following steps (Figure 1, both script and dataset available from https://osf.io/7b54w/): (1) Either 100, 400, 1600, or 6400 (i.e. four quadruplings) virtual subjects were allocated a true IQ score from a random normal distribution $(M=100, S D=15)$; (2) The virtual subjects were allocated a true creativity score from a random normal distribution $(M=100, S D=15)$ with a defined population correlation, drawn from random uniform distribution between 0 and 1, with the true IQ score; (3) The virtual subjects were allocated a disturbance score from a random beta distribution that was either negatively $($ alpha $=9$, beta $=1)$ or positively $($ alpha $=1$, beta $=9)$ skewed or approximately normally distributed $($ alpha $=9$, beta $=9)$. These disturbance scores varied between 0 and 1, with a low value indicating a high degree of disturbance; (4) The virtual subjects were allocated observed IQ and creativity scores by multiplying their true IQ and creativity scores with their disturbance score, i.e. the observed scores varied between zero and 
100 the true score. The present simulation did not assess the effect of measurement error other than

101 the influence of the defined disturbance variable.

102

103

104

105

106

107

108

109

110

111

112

113

114

115

116

117

118

119

120

121

122

\section{Defining verification}

The steps above were carried out 500 times for each of the 12 combinations of sample size and skewness of the disturbance variable. In these 6000 simulations, and following Jauk et al. (2013), the result was seen to support the threshold hypothesis if, and only if: (1) A significant breakpoint in the slope of the regression line was identified through segmented regression analysis (Muggeo, 2008); (2) The correlation between observed IQ and creativity was positive and significant below the breakpoint; (3) The correlation between observed IQ and creativity was significantly larger below compared to above the breakpoint. It should be noted that the threshold was not pre-defined, but rather empirically identified through segmented regression, again following the procedure by Jauk et al. (2013). Also in accordance with Jauk et al. and others, we did not demand for the correlation above the breakpoint to be non-significant. One of the reasons why this custom has evolved in threshold research might be to avoid encouraging researchers to limit the amount of data they collect, as a large sample would increase the likelihood for a small correlation above the threshold to become statistically significant.

\section{Analyses}

Analyses were carried out employing the segmented (version 0.5-3.0, Muggeo, 2008) and moments (version 0.14, Komsta \& Novometsky, 2015) packages in R. In segmented regression, the predicted outcome, $E|Y|$, is given by (Muggeo, 2008): 
125 In this equation, $\beta_{0}=$ predicted value on the outcome if the value on the predictor equals zero, i.e.

126 the intercept; $\beta_{1}=$ predicted increase in the outcome for an increase in the predictor by one, i.e.

127 the slope, given that the value on the predictor is below the tested breakpoint; $z_{i}=$ the value on

128 the predictor of individual $\mathrm{i} ; \beta_{2}=$ difference in the slope above and below the tested breakpoint;

$129 \psi=$ the tested breakpoint; $I=$ an indicator that equals one if the statement, i.e. $z_{i}>\psi$, is true and

130 zero otherwise. When conducting segmented regression with the segmented package in R, points

131 along the continuum of the predictor are tested and the point resulting in the largest absolute

132 value of $\beta_{2}$, with the additional requirement that the fitted lines should join at the breakpoint, is

133 identified and if $\beta_{2}$ differs significantly from zero a significant breakpoint has been found

134 (Muggeo, 2008).

135 For each of the 12 combinations of sample size and skewness of the disturbance variable, 136 logistic regression was used to predict the probability for a result in accordance with the 137 threshold hypothesis from the correlation between true IQ and true creativity.

If disturbance was not allowed to influence the association between true IQ/creativity and observed IQ/creativity, i.e. observed scores were identical to true scores, the predicted

142 probability for a result supporting the threshold hypothesis was lower than the $5 \%$ nominal type 143 I error rate (Figure 2). However, if disturbance was allowed to influence the association between 144 true IQ/creativity and observed IQ/creativity, a substantial probability to get a result in 
145 accordance with the threshold hypothesis could be observed, especially with a large sample size

146 and a weak correlation between true IQ and true creativity (Figure 3).

147 The effect of disturbance is illustrated in Figure 4, with no correlation between true IQ (M

$148=120, S D=15)$ and true creativity $(r=-.038, p=.453$, panel A). A segmented regression

149 analysis of the data in panel A indicated a breakpoint at true IQ $=163$, but the breakpoint was not

150 significant, i.e. there was no significant difference in the slope of the regression line below and

151 above the breakpoint $(p=.167)$, and the association is not in accordance with the threshold

152 hypothesis. However, if we assume variation in the degree of disturbance experienced by the

153 individuals, with some experiencing very high levels (indicated by a low value in panel B, the

154 values were drawn from a random beta distribution with alpha $=9$ and beta $=1$ ), and multiply

155 the individuals' true IQ and creativity scores with their experienced degree of disturbance, we

156 can observe a drift towards the lower left corner (panel C). Now we have an association that

157 could be seen to support the threshold hypothesis, with a general correlation between observed

158 IQ and observed creativity $(r=.306, p<.001)$, a significant breakpoint at observed IQ $=120(p$

$159=.020)$, and a significant positive correlation below the breakpoint $(r=.324, p<.001)$ that is

160 significantly stronger $(Z=3.633, p<.001)$ than the correlation above the breakpoint $(r=-.093, p$

$161=.363)$.

162 It is probably the individuals experiencing the highest degree of disturbance (i.e. those with

163 the lowest score on the disturbance variable), and consequently the largest south-western drift,

164 that are responsible for this phenomenon. If they are blessed with high true IQ and creativity

165 (black dots in panels A and C), their drift moves them into the crowded center, thereby

166 decreasing the correlation at the high end of the IQ scale. However, if they suffer from low true

167 IQ and creativity (snowflakes in panels A and C), their drift even further south-west will 
168 strengthen the correlation at the low end of the IQ scale. As observed IQ, observed creativity,

169 and residuals are quite nicely normally distributed (panels A-C in Figure 5) and because there is

170 no association between predicted degree of creativity and residuals from the segmented analysis

171 (panel D), with a mean residual very close to zero (the horizontal line in panel D) for every level

172 of predicted creativity, model diagnostics indicate a valid finding.

173

174

175

176

177

178

179

180

181

182

183

184

185

186

187

188

189

190

Discussion

The present simulation indicates that if subjects' observed scores on two variables that are not too strongly correlated, e.g. IQ and creativity, are simultaneously affected by some disturbing factor, e.g. low motivation, illness, or linguistic confusion, that varies in magnitude between subjects, there is a good chance of observing a threshold-like association between the two variables, at least if the analysis is not crippled by low power due to a small sample size.

We propose that such a possible influence of disturbance is a threat mainly against the validity of studies investigating threshold-like associations between abilities, e.g. IQ and creativity, that are operationalized as measured performances. The validity of such measures is not guaranteed, as lack of disturbance, together with high ability, is needed for a high performance, while presence of disturbance is enough for a low performance. Therefore, we could probably assume that the validity is positively correlated with measured performance and, consequently, tends to be lower among those with low scores. To paraphrase the threshold hypothesis: A high true intelligence/creativity is necessary, but not sufficient, for a high measured intelligence/creativity.

A consequence of the above thoughts might be a prediction that performances, generally, tend to have threshold-like associations with each other, as long as they are not perfect indicators 
191 of true abilities, the true abilities are not too strongly correlated with each other, and they are

192 simultaneously influenced, at least to some degree, by the same attenuating factors. For example,

193 decathletes' performances on pole vault and shot put might exhibit a threshold-like association

194 due to some of the athletes' mild illness, sore shoulder, or low motivation. Spearman's (1927)

195 "law of diminishing returns" reflects the observation that IQ test scores tend to be more highly

196 correlated among those with low, compared to those with high, ability (e.g. Deary et al., 1996).

197 Due to its similarity with the threshold hypothesis, both suggesting a stronger association at the

198 low end of the IQ scale, it is conceivable that both are due to the same cognitive mechanisms,

199 e.g. higher degree of differentiation of abilities at higher levels. However, yet another possibility

200 is that findings in accordance with Spearman's law are also, at least to some degree, due to an

201 influencing effect of disturbance.

202 As mentioned in the introduction, the threshold-like association between intelligence and

203 creativity has revealed itself in some studies but not in others. One, maybe the most likely,

204 explanation of this could be simple random variation. In the light of the present findings, another

205 possibility is that studies who have failed to detect this threshold have been less affected by

206 disturbing factors with a simultaneous influence on measured intelligence and creativity. We can

207 also note that the threshold-effect seems more elusive when creativity is measured as actual

208 achievement rather than as potential (Jauk et al., 2013). This difference seemingly fits with the

209 present findings, as high scores on tests of creative potential, for example trying to come up with

210 as many and as original alternate uses for a hairdryer as possible, are probably more reliant on

211 the absence of disturbing factors, e.g. low motivation, than are high scores on tests of creative

212 achievement, for example answering questions if you have produced something in the areas of

213 literature or creative cooking. 
Recently, a few studies have employed a newly developed method called Necessary

215 Condition Analysis (NCA, Dul, 2016) and claim to have found support for the notion that a

216

217

218

219

220

221

222

223

224

225

226

227

228

229

230

231

232

233

234

235

certain minimum level of intelligence is necessary for a high level of creativity (Karwowski et

al., 2016, 2017; Shi et al., 2017). However, as a negatively skewed predictor (e.g. measured

intelligence) and a positively skewed outcome (e.g. measured creativity) seems to be enough for

NCA to indicate a strong effect (Sorjonen, Wikström Alex, \& Melin, 2017), the validity of these

claims can also be called into question. Another way to evaluate the association between

intelligence and creativity could be to apply both non-linear and segmented linear models to

collected data and to compare the fit of these. This would give an indication if the association is

best described by a gradual (for example quadratic) or a sharp (segmented linear) change in the

slope. However, it is possible that in either case the observed association could still be due to an

influence of disturbance.

This study has some limitations. For example, for the disturbance variable we used beta

distributions (because these are bounded by 0 and 1, which seems logical for an attenuating

variable) that were either "substantially" positively or negatively skewed or approximately

normally distributed. Although the main result of this study did not depend on which of the three

distributions was used, there is of course a myriad of other possible distributions that could have

been employed, for example with extreme negative skewness (alpha $=100$, beta $=1)$, uniform

$($ alpha $=1$, beta $=1)$, or bimodal $($ alpha $=0.5$, beta $=0.5)$ distributions ${ }^{1}$. On balance, we believe

that a negatively skewed disturbance variable similar to the one in panel B in Figure 4 should be

quite realistic in many situations. It indicates that most subjects would get an observed IQ and

creativity score close to their true value (disturbance close to 1) while a few receive a score well

${ }^{1}$ If somebody happens to know the true distribution of the disturbance variable, they are welcome to implement this into the available script, or to inform us so that we can do it.

Peer] reviewing PDF | (2019:06:38518:2:0:NEW 6 Sep 2019) 
236 below their true value (only half of it in the most extreme case). Another limitation is that in each

237 simulation we used only one disturbance variable and this was common both for intelligence and

238 for creativity. In reality, there would probably be several different disturbance variables in play,

239 some of them common and some unique only for intelligence or for creativity.

240 Somebody might be tempted to argue that because this simulation has limitations, for

241 example that we do not know the exact distribution of the disturbance variable, its results are not

242 trustworthy and, therefore, (1) it does not disprove the intelligence-creativity threshold

243 hypothesis with absolute certainty, and (2) the intelligence-creativity threshold hypothesis must,

244 consequently, be considered correct. We admit that we cannot be sure that an effect of

245 disturbance is the sole or even one of the explanations of the intelligence-creativity threshold

246 association, demonstrated in various studies. However, we, as well as many others (e.g. Popper,

247 1959), would argue that "X has not been proven false with absolute certainty" does not

248 necessarily imply that " $\mathrm{X}$ is correct". We believe that the present simulation points at the

249 possibility that at least some of the findings verifying the threshold hypothesis could be due to an

250 influence of disturbance. It can also be noted that quite a few published studies, including the

251 meta-analysis by Kim (2005), found no support for the threshold hypothesis. This, together with

252 the high estimated rate of publication bias in psychological research (Ingre \& Nilsonne, 2018),

253 and the results of the present simulation, could be seen to indicate that there is, for the moment,

254 more speaking against than in favor of a true threshold-like association between intelligence and 255 creativity.

256

257 
259 Cho, S. H., Nijenhuis, J. T., van Vianen, A. E. M., Kim, H. -B., \& Lee, K. H. (2010). The relationship between diverse components of intelligence and creativity. The Journal of Creative Behavior, 44, 125-137.

262

263

264

265

266

267

268

269

270

271

272

Cummins, J. (1976) The influence of bilingualism on cognitive growth: A synthesis of research findings and explanatory hypotheses. Working Papers on Bilingualism, 9, 1-43.

Cummins, J. (1979) Linguistic interdependence and the educational development of bilingual children. Review of Educational Research, 49, 222-51.

Deary, I. J., Egan, V., Gibson, G. J., Austin, E. J., Brand, C. R., \& Kellaghan, T. (1996). Intelligence and the differentiation hypothesis. Intelligence, 23, 105-132.

Dul, J. (2016). Necessary Condition Analysis (NCA): Logic and methodology of "necessary but not sufficient" causality. Organizational Research Methods, 19, 10-52.

Fuchs-Beauchamp, K. D., Karnes, M. B., \& Johnson, L. J. (1993). Creativity and intelligence in preschoolers. Gifted Child Quarterly, 37, 113-117.

Guilford, J. P. (1967). The nature of human intelligence. New York: McGraw-Hill.

Ingre, M., \& Nilsonne, G. (2018). Estimating statistical power, posterior probability and publication bias of psychological research using the observed replication rate. Royal Society Open Science, 5, 181190.

Jauk, E., Benedek, M., Dunst, B., \& Neubauer, A. C. (2013). The relationship between intelligence and creativity: New support for the threshold hypothesis by means of empirical breakpoint detection. Intelligence, 41, 212-221. 
279 Karwowsi, M., Dul, J., Gralewski, J., Jauk, E., Jankowska, D. M., Gajda, A., Chruszczewski, M.

280 H., \& Benedek, M. (2016). Is creativity without intelligence possible? A necessary condition analysis. Intelligence, 57, 105-117.

282

283

284

285

286

287

288

289

290

291

292

293

294

295

296

297

298

299

300

Karwowski, M., Kaufman, J. C., Lebuda, I., Szumski, G., \& Firkowska-Mankiewicz, A. (2017). Intelligence in childhood and creative achievements in middle-age: The necessary condition approach. Intelligence, 64, 36-44.

Kim, K. H. (2005). Can only intelligent people be creative? A meta-analysis. The Journal of Secondary Gifted Education, 16, 57-66.

Komsta, L., \& Novomestky, F. (2015). moments: Moments, cumulants, skewness, kurtosis and related tests. R package version 0.14. https:/CRAN.R-project.org/package=moments

Max-Neef, M. (1995). Economic growth and quality of life: a threshold hypothesis. Ecological Economics, 15, 115-118.

Muggeo, V. M. R. (2008). segmented: an R Package to Fit Regression Models with BrokenLine Relationships. R News, 8/1, 20-25. URL http://cran.r-project.org/doc/Rnews/.

Popper, K. R. (1959). The logic of scientific discovery. London: Hutchinson.

Preckel, F., Holling, H., \& Wiese, M. (2006). Relationship of intelligence and creativity in gifted and non-gifted students: An investigation of threshold theory. Personality and Individual Differences, 40, 159-170.

Orians, G. H. (1969). On the evolution of mating systems in birds and mammals. The American Naturalist, 103, 589-603.

R Core Team (2018) R: A language and environment for statistical computing. R Foundation for Statistical Computing, Vienna, Austria. http://www.R-project.org 
301 Runco, M. A., \& Albert, R. S. (1986). The threshold theory regarding creativity and intelligence:

302 An empirical test with gifted and nongifted children. The Creative Child \& Adult 303 Quarterly, 11, 212-218.

304 Schubert, D. S. P. (1973). Intelligence as necessary but not sufficient for creativity. The Journal $305 \quad$ of Genetic Psychology, 122, 45-47.

306 Shi, B., Wang, L., Yang, J., Zhang, M., \& Xu, L. (2017). Relationship between divergent 307 thinking and intelligence: An empirical study of the threshold hypothesis with Chinese 308 children. Frontiers in Psychology, 8, 254.

309 Sligh, A. C., Conners, F. A., \& Roskos-Ewoldsen, B. (2005). Relation of creativity to fluid and crystallized intelligence. The Journal of Creative Behavior, 39, 123-136.

311 Sorjonen, K., Wikström Alex, J., \& Melin, B. (2017). Necessity as a function of skewness. Frontiers in Psychology, 8, 2192.

Spearman, C. (1927). The abilities of man. London: Macmillan. 
Figure 1. Illustration of the steps in the simulation (solid lines) and analyzed effect (dashed line).

Figure 2. P-values for the breakpoint according to segmented regression analysis (dots, black for

results that support the threshold hypothesis and grey for results that do not) and probability for a result supporting the threshold hypothesis (solid line) as a function of the correlation between true IQ and true creativity. The dashed line indicates $p=.05 .1500$ simulations with $N=6400$ in each.

Figure 3. P-values for the breakpoint according to segmented regression analysis (dots, black for result supporting the threshold hypothesis (solid line) as a function of the correlation between true IQ and true creativity, separately for the combinations of three ranges of skewness of the disturbance variable and four sample sizes. The dashed line indicates $p=.05$. Approximately 500 simulations in each panel.

Figure 4. When two non-associated variables (e.g. intelligence and creativity, panel A) are both multiplied with the same attenuating variable (e.g. motivation) that varies in magnitude between individuals (panel B, with low scores indicating high degree of disturbance, values have been drawn from a random beta distribution with alpha $=9$ and beta $=1)$, we get two variables with a threshold-like association ( panel C). Black dots = persons with high true IQ, high true creativity, and high degree of disturbance; Snowflakes = persons with low true IQ, low true creativity, and 
339 high degree of disturbance. $N=400$. The line with a small dot at the bottom in panels A and C 340 indicates the empirically calculated breakpoint with a 95\% CI.

341

342 Figure 5. The frequency distribution of observed IQ (panel A), observed creativity (panel B), 343 and residuals (= difference between observed and predicted creativity according to the 344 segmented analysis, panel C) as well as the association between predicted creativity and 345 residuals (panel D).

346 


\section{Figure 1}

Steps in the simulation and analysis.

Illustration of the steps in the simulation (solid lines) and analyzed effect (dashed line). 


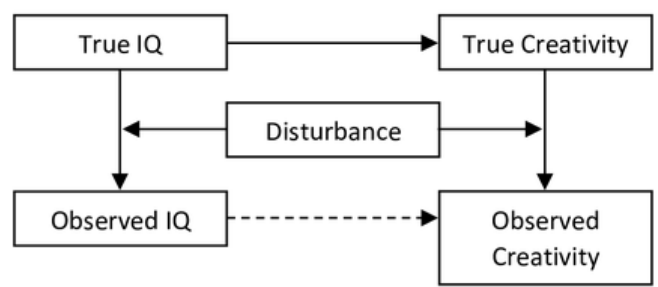




\section{Figure 2}

Association with no effect of disturbance.

P-values for the breakpoint according to segmented regression analysis (dots, black for results that support the threshold hypothesis and grey for results that do not) and probability for a result supporting the threshold hypothesis (solid line) as a function of the correlation between true IQ and true creativity. The dashed line indicates $p=.05 .1500$ simulations with $N=6400$ in each. 


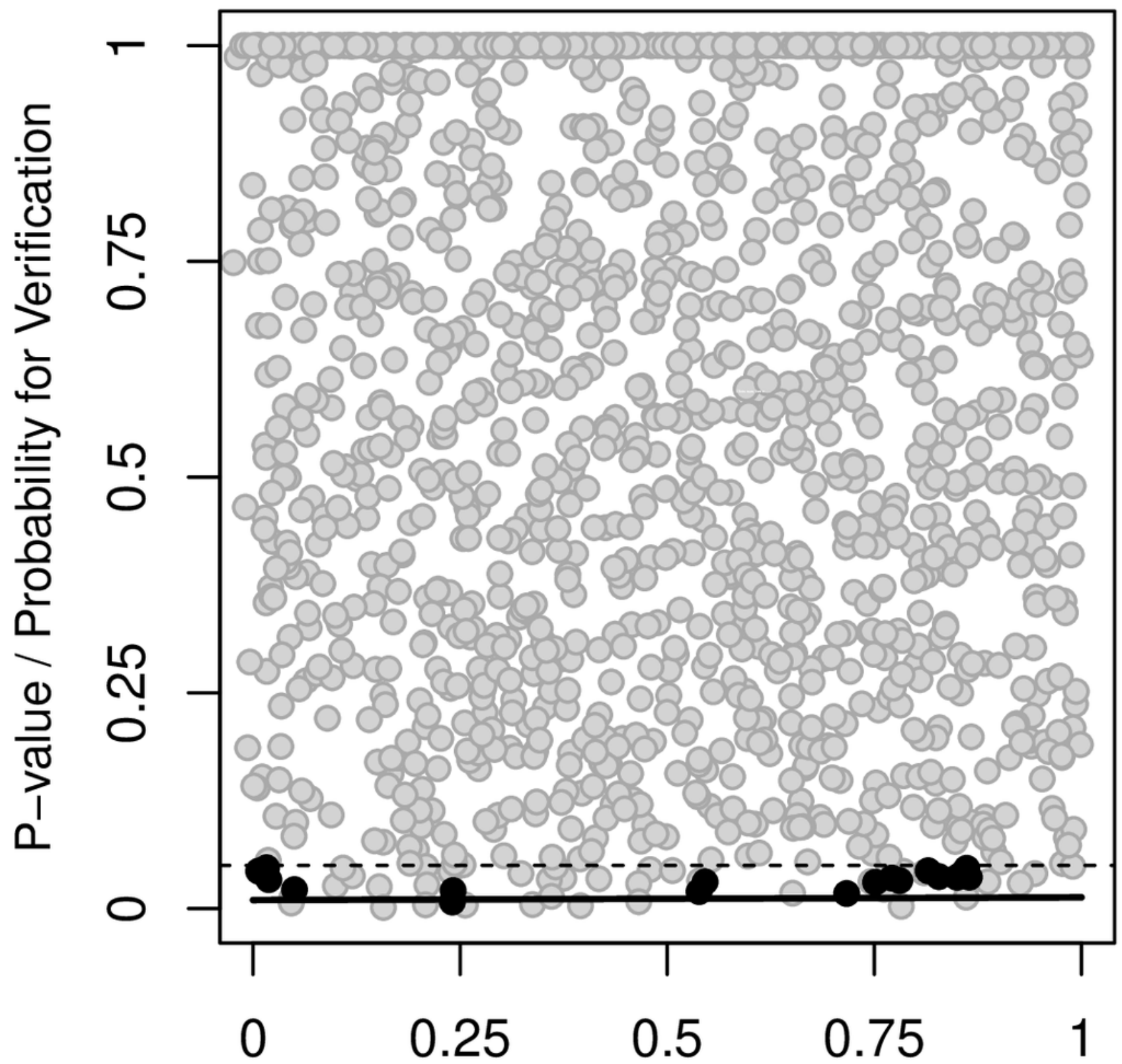

Correlation between true IQ and true creativity 


\section{Figure 3}

Association with an effect of disturbance.

P-values for the breakpoint according to segmented regression analysis (dots, black for results that support the threshold hypothesis and grey for results that do not) and probability for a result supporting the threshold hypothesis (solid line) as a function of the correlation between true IQ and true creativity, separately for the combinations of three ranges of skewness of the disturbance variable and four sample sizes. The dashed line indicates $p=$ .05. Approximately 500 simulations in each panel. 


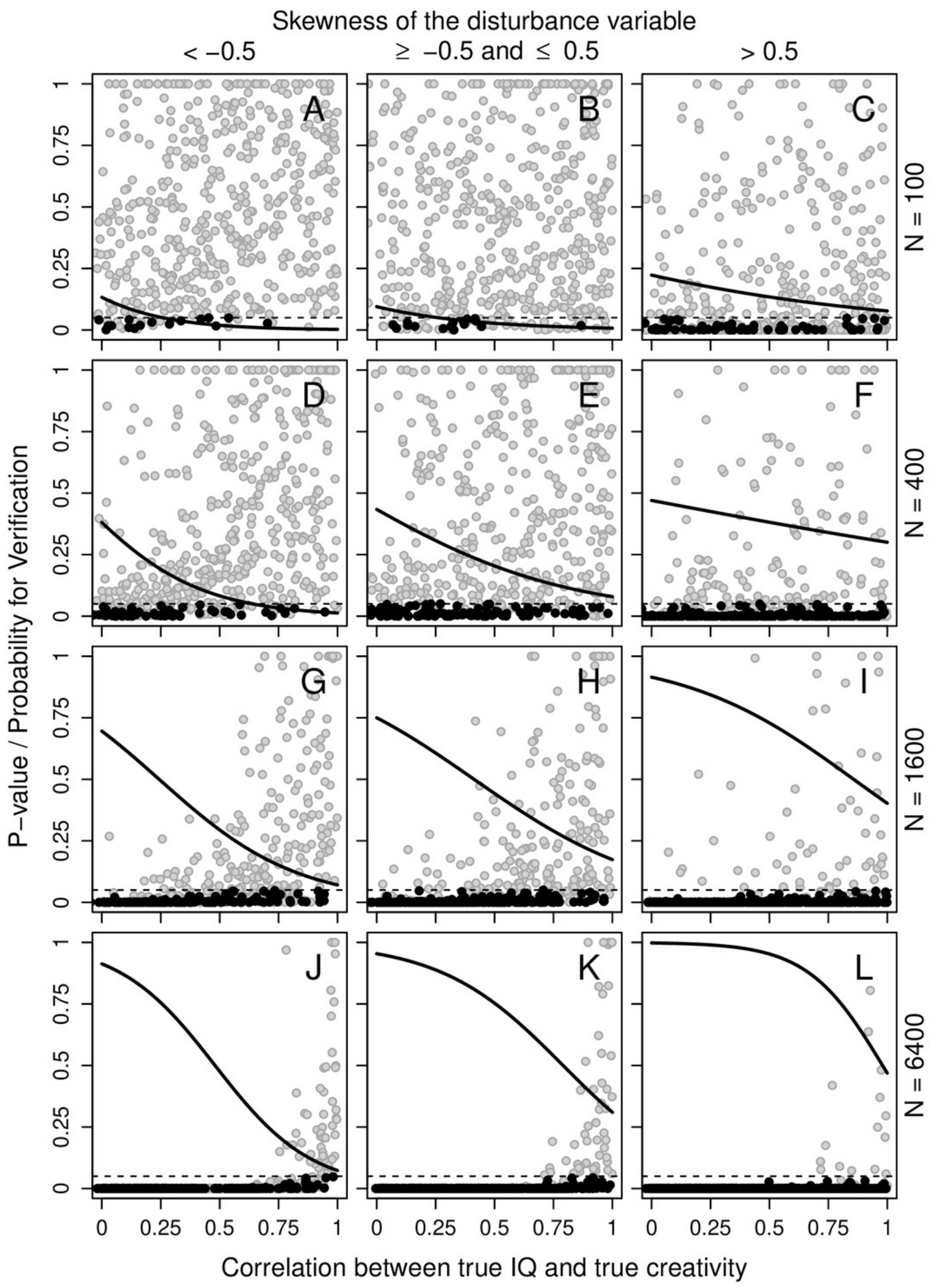


Figure 4

Example of the influence of disturbance.

When two non-associated variables (e.g. intelligence and creativity, panel A) are both multiplied with the same attenuating variable (e.g. motivation) that varies in magnitude between individuals (panel B, with low scores indicating high degree of disturbance, values have been drawn from a random beta distribution with alpha $=9$ and beta $=1$ ), we get two variables with a threshold-like association (panel C). Black dots = persons with high true IQ, high true creativity, and high degree of disturbance; Snowflakes = persons with low true IQ, low true creativity, and high degree of disturbance. $N=400$. The line with a small dot at the bottom in panels $\mathrm{A}$ and $\mathrm{C}$ indicates the empirically calculated breakpoint with a $95 \% \mathrm{Cl}$.
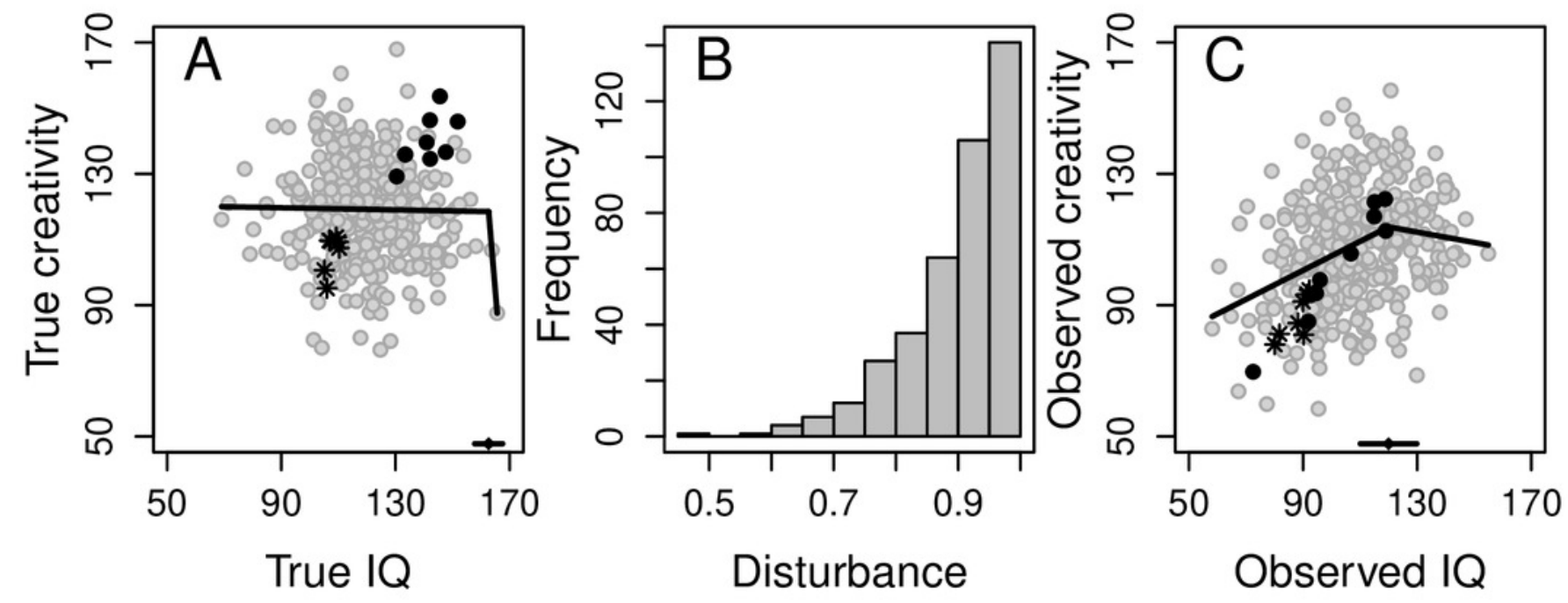
Figure 5

Diagnostics of segmented analysis.

The frequency distribution of observed IQ (panel A), observed creativity (panel B), and residuals ( $=$ difference between observed and predicted creativity according to the segmented analysis, panel C) as well as the association between predicted creativity and residuals (panel $D$ ).
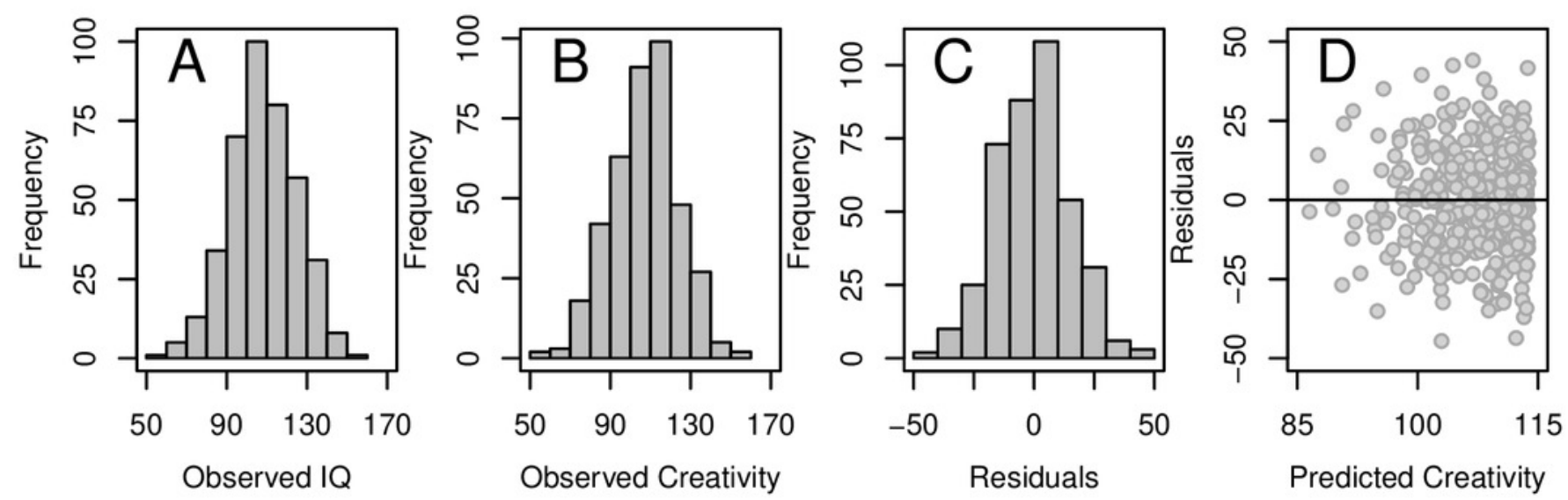